\title{
A SEMI-STRONG MINIMUM FOR A MULTIPLE INTEGRAL PROBLEM IN THE CALCULUS OF VARIATIONS
}

\author{
BY \\ WILLIAM KARUSH
}

Introduction. We consider an integral of the form

$$
I(S)=\int_{A} f\left(x^{1}, \cdots, x^{n}, z, p^{1}, \cdots, p^{n}\right) d x^{1} \cdots d x^{n}
$$

defined for a class of $n$-dimensional surfaces $S: z=z\left(x^{1}, \cdots, x^{n}\right)$ in $(n+1)$ space. The integral $I(S)$ is to be evaluated by setting

$$
z=z\left(x^{1}, \cdots, x^{n}\right), \quad p^{i}=\frac{\partial z}{\partial x^{i}} \quad(i=1,2, \cdots, n)
$$

and integrating over the fixed domain $A$. We seek sufficient conditions on a surface $S_{0}$ which will ensure that $S_{0}$ provides $I(S)$ with a relative ininimum in the class of surfaces $S$ which coincide with $S_{0}$ on the boundary $C$ of $A$.

In 1917 Lichtenstein [5] ( $\left.{ }^{1}\right)$ considered the case $n=2$ and by constructing a field established a sufficiency theorem for a strong relative minimum. He supposed analyticity for the functions involved, and assumed for the second variation $I_{2}$ a Jacobi condition expressed in terms of the characteristic values of a boundary value problem associated with the accessory partial differential equation. In the present paper we prove, without field theory and for the case of general $n$, a sufficiency theorem for a semi-strong relative minimum under much less stringent analytic requirement. We also give an estimate of the difference $I(S)-I\left(S_{0}\right)$. We assume for the second variation a condition of the form

$$
I_{2}(\zeta) \geqq \gamma \int_{A} \zeta^{2} d x^{1} \cdots d x^{n},
$$

where $\gamma$ is a positive constant, and $\zeta$ is any variation vanishing on $C$. Reid [10] has announced, but not yet published, a sufficiency theorem for a strong relative minimum for multiple integrals.

Few sufficiency theorems for multiple integrals are known. The main interest in the present paper lies in the method, which is indirect and appears to hold promise for extension to other problems. The indirect method of proof has been recently applied to simple integral problems by McShane [6],

Presented to the Society, August 23, 1946; received by the editors, June 21, 1946, and, in revised form, March 4, 1947.

(1) Numbers in brackets refer to the list of references at the end of the paper. 
Myers [8], and Hestenes [4].

Section 1 is devoted to a formulation of the problem. In section 2 we prove a convergence theorem closely related to the work of Morrey [7]. In section 3 we derive some properties of the Weierstrass $E$-function, and in section 4 we make the proof of the main theorem.

1. Formulation of problem. We write $x$ for the vector $\left(x^{1}, \cdots, x^{n}\right), p$ for $\left(p^{1}, \cdots, p^{n}\right), d x$ for $d x^{1} \cdots d x^{n}$, and adopt the convention that a repeated index means summation over that index. We remark that $z$ does not represent a vector.

The integrand function $f(x, z, p)$ is taken to be defined and of class $C^{\prime \prime}$ on an open set in $(x, z, p)$-space, and the integral $I(S)$ is to be understood in the Lebesgue sense. A point $(x, z, p)$ will be called admissible in case it lies in the domain of definition of $f$.

The region $A$ is taken to be a bounded, open, connected set in $n$-space with boundary denoted by $C$. The closure $A+C$ of $A$ will be denoted by $\bar{A}$; we do not assume that the $n$-dimensional Lebesgue measure of $C$ is zero. We point out that the domain of integration in $I(S)$ is the open set $A$.

The surfaces $S$ we shall consider are given in the form $z=z(x)$, where $z(x)$ is defined and Lipschitzian on $\bar{A}$; that is, there exists a constant $B$ such that $\left|z\left(x_{1}\right)-z(x)\right| \leqq B\left[\left(x_{1}^{i}-x^{i}\right)\left(x_{1}^{i}-x^{i}\right)\right]^{1 / 2}$ for $x_{1}$ and $x$ in $\bar{A}$. For such a function the partial derivatives $\partial z / \partial x^{i}$ exist almost everywhere on $A$, and $\left|\partial z / \partial x^{i}\right|^{\alpha}$ is summable on $A$ for arbitrary $\alpha>0$. We shall of ten use $p^{i}$ to represent the derivatives of $z$. A surface $S: z=z(x)$ will be called admissible in case it satisfies the following conditions: (i) $z(x)$ is Lipschitzian on $\bar{A}$; (ii) $(x, z, p)$ is admissible whenever $x$ is in $A$ and all the partial derivatives $p^{i}=\partial z / \partial x^{i}$ exist; (iii) the integral $I(S)$ exists, finite or infinite.

For any function $h$ in $L_{2}$ on $A$, that is, integrable square on $A$, we define

$$
\|h\|^{2}=\int_{A} h^{2} d x .
$$

We call an admissible surface $S: z=z(x)$ a surface of class $C^{\prime}$ in case $z\left(x_{-}\right)$ can be extended to a neighborhood of $\bar{A}$ in such a way that the extended function is of class $C^{\prime}$, in the usual sense, in the neighborhood. Whenever reference is made to such a surface without specific designation of the range of $x$ it is understood that the range of $x$ is $\bar{A}$. An extremaloid $S_{0}: z=z_{0}(x)$ is an admissible surface of class $C^{\prime}$ which satisfies the Haar-Coral condition. This condition asserts that for every $n$-dimensional cube $R$ which together with its boundary $R^{*}$ lies in $A$, the relation

$$
\int_{R} f_{z} d x=\int_{R^{*}} f_{p^{i}} \nu^{i} d s
$$

holds, where $\nu=\left(\nu^{1}, \cdots, \nu^{n}\right)$ is the outer normal to $R^{*}, d s$ is the $(n-1)$ - 
dimensional element of area on $R^{*}$, and the partial derivatives of $f$ are evaluated at $S_{0}$. A surface $S_{0}$ is called nonsingular in case the determinant $\left|f_{p^{i}} p^{i}\right|$ is different from zero at every point of $S_{0}$.

Associated with the integral $I$ is the Weierstrass E-function

$$
E(x, z, p, P) \equiv f(x, z, P)-f(x, z, p)-\left(P^{i}-p^{i}\right) f_{p^{i}}(x, z, p) .
$$

Let $S_{0}$ be an admissible surface of class $C^{\prime}$. We shall say that $S_{0}: z=z_{0}(x)$ satisfies the Weierstrass condition $\mathrm{II}_{N ; M}$ with constant $M>0$ in case the following properties are satisfied( $\left.{ }^{2}\right)$ : (i) $p_{0}^{4}(x) p_{0}^{1}(x)<M$ for $x$ in $\bar{A}$, where the $p_{0}^{1}$ are the partial derivatives of $z_{0}$; (ii) there exists a neighborhood $N$ in $(x, z, p)$ space of the values $\left(x, z_{0}, p_{0}\right)$ belonging to $S_{0}$ such that the inequality

$$
E(x, z, p, P) \geqq 0
$$

holds for $(x, z, p)$ in $N,(x, z, P)$ admissible with $P^{i} P^{i}<M$. For $M=+\infty$ this condition reduces to the standard Weierstrass condition $\mathrm{II}_{N}$. We shall have occasion to deal only with the case $M$ finite.

By an admissible variation we shall mean a function $\zeta(x)$ which is defined and Lipschitzian on $\bar{A}$, and vanishes on $C$. The first variation $I_{1}(\zeta)$ of the integral $I$ along the surface $S_{0}$ is given by

$$
I_{1}(\zeta)=\int_{A}\left(f_{z} \zeta+f_{p^{i}} \pi^{i}\right) d x
$$

where $\pi^{i}=\zeta_{x^{i}}$, and $f_{z}, f_{p^{i}}$ are evaluated at $S_{0}$. It may be shown that a surface of class $C^{\prime}$ is an extremaloid if and only if the first variation along $S_{0}$ vanishes for every admissible variation. The proof will be omitted. It may be made by the method of Carson [2], with a modification consisting in the use of a device employed by Reid [9, proof of Lemma 2.2] to take care of the irregularity of the boundary $C$.

The second variation $I_{2}(\zeta)$ of $I$ along $S_{0}$ is given by

$$
I_{2}(\zeta)=\int_{A} 2 \omega\left(x ; \zeta, \zeta_{x^{i}}\right) d x
$$

where

$$
2 \omega\left(x ; \zeta, \pi^{i}\right)=f_{z z} \zeta^{2}+2 f_{z p^{i}} \zeta \pi^{i}+f_{p^{i} p^{i} \pi^{i} \pi^{j}}
$$

and the partial derivatives of $f$ are evaluated along $S_{0}$. We shall say that a surface $S_{0}$ satisfies the strengthened Jacobi condition in case there exists a positive constant $\gamma$ such that

$$
I_{2}(\zeta) \geqq \gamma\|\zeta\|^{2}
$$
tion.

(2) The author is indebted to the referee for suggesting this form of the Weierstrass condi- 
for every admissible variation $\zeta$. This inequality is a strengthening of the well known necessary condition $I_{2}(\zeta) \geqq 0$ for a minimizing surface, but is not the only form of a strengthened Jacobi condition that may be considered. An important necessary condition for a minimizing surface is the one involving the nature of the solutions of the Haar-Coral equation for the second variation [9]. One way of strengthening this necessary condition is to assume that there exists a solution of the Haar-Coral equation for the second variation which is everywhere different from zero. It may be shown that such a strengthened Jacobi condition implies our strengthened condition. We shall not make use of this result. For simple integral problems the two strengthened conditions are equivalent.

Our main theorem is the following.

THEOREM. Let $S_{0}: z=z_{0}(x)$ be a nonsingular extremaloid which satisfies the strengthened Jacobi condition. Let $M>0$ be any finite constant with which $S_{0}$ satisfies the Weierstrass condition $\mathrm{II}_{N ; M}$. Then there exists an $\epsilon>0$ and a neighborhood $\mathcal{F}$ of $S_{0}$ in $(x, z)$-space such that the inequality

$$
I(S)-I\left(S_{0}\right)>\min \left\{\epsilon, \epsilon\left(\left\|z-z_{0}\right\|^{2}+\left\|p^{i}-p_{0}^{i}\right\| \cdot\left\|p^{i}-p_{0}^{i}\right\|\right)\right\}
$$

holds for any admissible surface $S: z=z(x)$ distinct from $S_{0}$ which lies in $\mathcal{F}$, coincides with $S_{0}$ on the boundary $C$, and satisfies $p^{i} p^{i}<M$ for almost all $x$ in $A$.

2. A convergence theorem. The convergence theorem stated below may be deduced from the extensive results of Morrey [7]. For completeness we present a short proof of a result sufficient for our needs.

If a function $u(x)$ is Lipschitzian on $\bar{A}$ then $u$ has partial derivatives almost everywhere in $A$. If, in addition, $u$ vanishes on $C$ we may define its partial derivatives almost everywhere on $C$ by extending $u$ to be zero on the complement of $\bar{A}$ and using the derivatives of the extended function. It is in this sense that we shall interpret partial derivatives on the boundary $C$ for a function which vanishes on $C$. By writing $u$ as the difference of two nonnegative Lipschitzian functions it is easy to show [9, Lemma 2.1] that the partial derivatives $u_{x^{i}}$ vanish almost everywhere on $C$.

THEOREM 2.1. Let $\left\{u_{\alpha}\right\}$ be a sequence of admissible variations with

$$
\int_{A}\left(u_{\alpha}^{2}+\pi_{\alpha}^{i} \pi_{\alpha}^{i}\right) d x<\text { const. } \quad(\alpha=1,2, \ldots ; \alpha \text { not summed }),
$$

where $\pi_{\alpha}^{i}=u_{\alpha x}$. Then there exists a subsequence $\left\{u_{\beta}\right\}$, and $n+1$ functions $u_{0}, \pi_{0}^{s}$ each in $L_{2}$ on $\bar{A}$ with the following properties:

$$
\int_{0}\left[U(x) u_{\beta}+V^{i}(x) \pi_{\beta}^{i}\right] d x \rightarrow \int_{0}\left[U(x) u_{0}+V^{i}(x) \pi_{0}^{i}\right] d x \quad \text { as } \beta \rightarrow \infty
$$


for $U, V^{i}$ arbitrary functions in $L_{2}$ on $\bar{A}$ and $e$ an arbitrary measurable subset of $\bar{A}$;

$$
\int_{\bar{\Lambda}}\left(u_{\beta}-u_{0}\right)^{2} d x \rightarrow 0 \quad \text { as } \beta \rightarrow \infty ;
$$

(iii) there exists a sequence $\left\{v_{\beta}\right\}$ of admissible variations such that

$$
\left.\int_{\bar{A}}\left[\left(v_{\beta}-u_{0}\right)^{2}+\left(\alpha_{\beta}^{i}-\pi_{0}^{i}\right)\left(\kappa_{\beta}^{i}-\pi_{0}^{i}\right)\right] d x \rightarrow 0 \quad \text { as } \beta \rightarrow \infty \text { ( } \beta \text { not summed }\right),
$$

where $\kappa_{\beta}^{i}=v_{\beta x^{i}}$.

In the proof we shall use freely concepts and results from the theory of Banach spaces. We denote by $B$ the Banach space of all functions $g$ in $L_{2}$ on $\bar{A}$ with norm

$$
\|g\|_{1}=\left(\int_{\bar{A}} g^{2} d x\right)^{1 / 2}
$$

Observe that this norm differs from the norm $\|g\|$ introduced in the previous section in having $\bar{A}$ for the domain of integration instead of $A$. Let $\mathbb{B}_{n}$ be the Banach space of all sets $\left(g_{i}^{0}, g^{1}, \cdots, g^{n}\right)$ of $n+1$ functions in $L_{2}$ on $\bar{A}$ with norm

$$
\left\|\left(g^{0}, g^{1}, \cdots, g^{n}\right)\right\|_{1}=\left\|g^{0}\right\|_{1}+\left\|g^{1}\right\|_{1}+\cdots+\left\|g^{n}\right\|_{1} .
$$

Observe that since $u_{\alpha}=\pi_{\alpha}^{s}=0$ almost everywhere on $C$ we have

$$
\left\|\left(u_{\alpha}, \pi_{\alpha}\right)\right\|_{1}^{2} \equiv\left\|\left(u_{\alpha}, \pi_{\alpha}^{1}, \cdots, \pi_{\alpha}^{n}\right)\right\|_{1}^{2}<\text { const. } \quad(\alpha=1,2, \cdots) .
$$

We first establish a lemma.

LeMma 2.1. Let $\left\{u_{\alpha}\right\}$ satisfy the hypotheses of the above theorem. Then there exists a subsequence $\left\{u_{\beta}\right\}$ and a function $u_{0}$ in $\mathbb{B}$ such that

$$
\left\|u_{\beta}-u_{0}\right\|_{1} \rightarrow 0 \quad \text { as } \beta \rightarrow \infty .
$$

It is sufficient to prove the lemma for $A$ a hypercube, since we may extend each $u_{\alpha}$ to be zero outside of $\bar{A}$ and then consider the sequence $\left\{u_{\alpha}\right\}$ in a large hypercube containing $\bar{A}$. From the relation (2.1) we see that the sequence $\left\{u_{\alpha}\right\}$ will satisfy the appropriate norm inequality in the hypercube. For the details of the proof in this case we refer to Theorem 8.3 and Lemma 8.3 of Morrey [7].

To prove the theorem we suppose that a subsequence of $\left\{u_{\alpha}\right\}$ has been selected according to the lemma which converges in norm in $B$ to some function $\bar{u}_{0}$ in $\mathscr{B}$. We retain the same notation for the subsequence. In $\mathscr{B}_{n}$ the sequence $\left(u_{\alpha}, \pi_{\alpha}^{i}\right)(\alpha=1,2, \cdots)$ has uniformly bounded norm by the 
inequality (2.1). It follows from well known results on weak compactness in Banach spaces $\left[1\right.$, chap. 8] that there exists an element $\left(u_{0}, \pi_{0}\right)=\left(u_{0}, \pi_{0}^{1}\right.$, $\left.\cdots, \pi_{0}^{n}\right)$ in $B_{n}$ which is the weak limit of a subsequence $\left(u_{\beta}, \pi_{\beta}\right)$. Since

$$
\int_{0}\left(U g^{0}+V^{i} g^{i}\right) d x
$$

is a continuous additive functional on $\mathscr{B}_{n}$, conclusion (i) of the theorem follows. Clearly $\left\{u_{\beta}\right\}$ converges weakly to $u_{0}$ in $B$. Since $\left\{u_{\beta}\right\}$ converges strongly to $\bar{u}_{0}$ in $\mathcal{B}$, we obtain $u_{0}=\bar{u}_{0}$ and conclusion (ii) holds. From another theorem on Banach spaces [1, chap. 9] we may assert that there exists a sequence of finite linear combinations of $\left\{u_{\beta}, \pi_{\beta}\right\}$ which converges in norm in $\mathscr{B}_{n}$ to $\left(u_{0}, \pi_{0}\right)$. This sequence must have the form $\left(v_{\beta}, \kappa_{\beta}\right)$ with $v_{\beta}$ Lipschitzian on $\bar{A}$, $\kappa_{\beta}^{*}=v_{\beta x t}$, and $v_{\beta}=0$ on $C$ since $\left\{\left(u_{\beta}, \pi_{\beta}\right)\right\}$ has these properties. This completes the proof.

3. The $E$-function. In the present section we let $S_{0}: z=z_{0}(x)$ be an admissible surface of class $C^{\prime}$, and write $p_{0}^{i}=z_{0 x^{\prime}}(i=1,2, \cdots, n)$. We let $N$, with or without subscripts, denote a neighborhood in $(x, z, p)$-space of the values $\left(x, z_{0}, p_{0}\right)$ belonging to $S_{0}$ (on $\bar{A}$ ), and shall suppose that such a neighborhood is restricted to contain only admissible elements. If $S_{0}$ satisfies the condition $\mathrm{II}_{N ; M}$ we may assume also that each neighborhood $N_{1}$ discussed below is contained in $N$, where $N$ is the neighborhood for which $\mathrm{II}_{N ; M}$ is effective, and that $p^{i} p^{i}<M$ holds for $(x, z, p)$ in $N_{1}$.

LEMMA 3.1. Let $S_{0}$ be a nonsingular surface which satisfies condition $\mathrm{II}_{N_{;} M}$ with a finite constant $M>0$. Then there exists a constant $\lambda_{1}>0$ and a neighborhood $N_{1}$ such that the inequality

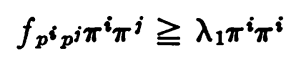

holds for $\pi$ arbitrary, and $(x, z, p)$ in $N_{1}$.

This is a well known result in the calculus of variations.

Lemma 3.2. If $S_{0}$ satisfies the hypotheses of Lemma 3.1 then there exists a constant $\lambda_{2}>0$ and $a$ neighborhood $N_{2}$ such that the inequality

$$
E(x, z, p, P) \geqq \lambda_{2}\left(P^{i}-p^{i}\right)\left(P^{i}-p^{i}\right)
$$

holds for $(x, z, p),(x, z, P)$ in $N_{2}$.

This lemma follows immediately from Lemma 3.1 by Taylor's expansion theorem.

Lemma 3.3. Let $S_{0}$ satisfy the hypotheses of Lemma 3.1. Then for any neighborhood $N_{3}$ there exists a constant $\lambda>0$ and a neighborhood $N$ contained in $N_{3}$ such that the inequality

$$
E(x, z, p, P) \geqq \lambda\left(P^{i}-p^{i}\right)\left(P^{i}-p^{i}\right)
$$


holds for $(x, z, p)$ in $N,(x, z, P)$ admissible and outside $N_{3}$ with $P^{i} P^{i}<M$.

Proof. Select $N_{0} \subset N_{3}$ such that if $(x, z, p),(x, z, p+\pi)$ are in $N_{0}$ then $(x, z, p+\theta \pi)$ lies in the neighborhood $N_{1}$ of Lemma 3.1 for $0 \leqq \theta \leqq 1$. Select $N$ so that $\bar{N} \subset N_{0}$. We shall show that $N$ is effective in the lemma. There exists a constant $k>0$ such that if $(x, z, p)$ belongs to $N$ and $\pi^{i} \pi^{i}<4 k^{2}$, then $(x, z, p+\pi)$ belongs to $N_{0}$. Let $(x, z, p),(x, z, P)$ be as in the lemma. We write $P=p+h \pi$, with $\pi^{i} \pi^{i}=k^{2}$ and $h \geqq 0$. Since $(x, z, P)$ does not belong to $N_{0}$ we have $h \geqq 2$. On the other hand, since $P^{i} P^{i}<M$ and $M$ is finite, we have $h<H$ where $H$ is a constant depending only on $k$ and $M$.

By Taylor's expansion theorem, and condition $\mathrm{II}_{N_{;} M}$, we obtain (suppressing the $x, z$ arguments)

$$
E(p+\pi, P)=E(p, P)+\pi^{i} E_{p^{i}}(p+\theta \pi, P) \geqq 0 \quad(0<\theta<1) .
$$

Calculation of the partial derivatives of $E$ and Lemma 3.1 yields

$$
\begin{aligned}
E(p, P) & \geqq(h-\theta) \pi^{i} \pi^{i} f_{p^{i} p^{i}}(p+\theta \pi) \\
& \geqq(2-\theta) \lambda_{1} \pi^{i} \pi^{i} \\
& \geqq\left(\lambda_{1} / H^{2}\right)\left(P^{i}-p^{i}\right)\left(P^{i}-p^{i}\right) .
\end{aligned}
$$

Combining Lemmas 3.3 and 3.2 we may deduce the following result.

Lemma 3.4. Let $S_{0}$ satisfy the hypotheses of Lemma 3.1. Then there exists a constant $\lambda>0$ and $a$ neighborhood $N_{0}$ such that the inequality

$$
E(x, z, p, P) \geqq \lambda\left(P^{i}-p^{i}\right)\left(P^{i}-p^{i}\right)
$$

holds for $(x, z, p)$ in $N_{0}$, and $(x, z, P)$ admissible with $P^{i} P^{i}<M$.

We denote by $A(S)$ the area of $S$ over $A$,

$$
\mathcal{A}(S) \equiv \int_{A}\left(1+p^{i} p^{i}\right)^{1 / 2} d x
$$

Lemma 3.5. Let $H^{*}(S)$ be an integral of the form

$$
H^{*}(S)=\int_{A}\left[U(x, z)+p^{i} V^{i}(x, z)\right] d x,
$$

where $U, V^{i}$ are defined and continuous in a neighborhood of $S_{0}$ in $(x, z)$-space. Then for any $K>0$ and $\epsilon>0$ there exists a neighborhood $\mathcal{F}$ of $S_{0}$ in $(x, z)$-space such that the inequality

$$
\left|H^{*}(S)-H^{*}\left(S_{0}\right)\right|<\epsilon
$$

holds for any admissible surface $S$ in $\mathcal{F}$ which coincides with $S_{0}$ on $C$ and has $\mathcal{A}(S) \leqq K$.

Proof. Let $U_{0}(x) \equiv U\left(x, z_{0}(x)\right), V_{0}^{i}(x) \equiv V^{i}\left(x, z_{0}(x)\right)$. The functions $V_{0}^{i}$ are 
continuous on $\bar{A}$ and hence, by the Weierstrass approximation theorem, for each $i$ there exists a sequence of polynomials $\left\{V_{\alpha}^{i}(x)\right\}(\alpha=1,2, \cdots)$ which approaches $V_{0}^{t}$ uniformly on $\bar{A}$ as $\alpha \rightarrow \infty$. For any surface $S$, let

$$
F(S) \equiv \max _{x \operatorname{in} \bar{A}}\left\{\left[U(x, z(x))-U_{0}(x)\right]^{2}+\left[V^{i}(x, z(x))-V_{0}^{i}\right]\left[V^{i}-V_{0}^{i}\right]\right\}^{1 / 2}
$$

We also define

$$
\begin{aligned}
& G_{\alpha} \equiv \max _{x \text { in } A}\left[\left(V_{0}^{i}-V_{\alpha}^{i}\right)\left(V_{0}^{i}-V_{\alpha}^{i}\right)\right]^{1 / 2} \quad(\alpha \text { not summed }), \\
& F_{\alpha} \equiv \max _{x \text { in } \bar{A}}\left|V_{\alpha x^{1}}+V_{\alpha x^{2}}+\cdots+V_{\alpha x^{n}}\right| \text {. }
\end{aligned}
$$

We have

$$
\begin{aligned}
\mid H^{*}(S)- & H^{*}\left(S_{0}\right) \mid \\
\leqq & \left|\int_{A}\left[\left\{U(x, z(x))-U_{0}(x)\right\}+p^{i}\left\{V^{i}(x, z(x))-V_{0}^{i}(x)\right\}\right] d x\right| \\
& \quad+\left|\int_{A}\left(p^{i}-p_{0}^{i}\right)\left(V_{0}^{i}-V_{\alpha}^{i}\right) d x\right|+\left|\int_{A}\left(p^{i}-p_{0}^{i}\right) V_{\alpha}^{i} d x\right| \\
\leqq & A(S) F(S)+\left[\mathcal{A}(S)+\mathcal{A}\left(S_{0}\right)\right] G_{\alpha}+\left|\int_{A}\left(p^{i}-p_{0}^{i}\right) V_{\alpha}^{i} d x\right| .
\end{aligned}
$$

It remains to obtain an estimate of the last integral. To this end we observe that since $z_{0}(x)$ is of class $C^{\prime}$ in a neighborhood of $\bar{A}$ it may be extended to all of $x$-space in such a way as to remain of class $C^{\prime}$ in the whole space [3]. We retain the same notation for the extended function. For any surface $S$ which coincides with $S_{0}$ on $C$ we define $z(x) \equiv z_{0}(x)$ outside of $\bar{A}$. Then $z$ is Lipschitzian in any bounded region in space, and from the introductory remarks in the second paragraph of $\$ 2$ we see that $p^{i}=p_{0}^{i}$ almost everywhere outside $A$. Thus if $R$ is an open hyper-cube containing $\bar{A}$,

$$
\begin{aligned}
\left|\int_{A}\left(p^{i}-p_{0}^{i}\right) V_{\alpha}^{i} d x\right| & =\left|\int_{R}\left(p^{i}-p_{0}^{i}\right) V_{\alpha}^{i} d x\right| \\
& =\left|\int_{R}\left(V_{\alpha x^{1}}+V_{\alpha x^{2}}+\cdots+V_{\alpha x^{n}}\right)\left(z-z_{0}\right) d x\right| \\
& =\left|\int_{A}\left(V_{\alpha x^{1}}+V_{\alpha x^{2}}+\cdots+V_{\alpha x^{n}}\right)\left(z-z_{0}\right) d x\right| \\
& \leqq m F_{\alpha} \max _{x \text { in } \bar{A}}\left|z-z_{0}\right|
\end{aligned}
$$

where $m=$ meas $(A)$. The third integral was obtained by Fubini's theorem 
and integration by parts. With this inequality and the previous one we can complete the proof. For, given an $\epsilon>0$ we select $\alpha_{0}$ so that $\left[\mathcal{A}(S)+\mathcal{A}\left(S_{0}\right)\right] G_{\alpha_{0}}$ $\leqq\left[K+\mathcal{A}\left(S_{0}\right)\right] G_{\alpha_{0}}<\epsilon / 3$; then we determine $\mathcal{F}$ so that $m F_{\alpha_{0}} \max \left|z-z_{0}\right|$ $<\epsilon / 3$, and $\mathcal{A}(S) F(S) \leqq K F(S)<\epsilon / 3$. This proves the theorem.

For any admissible surface $S: z=z(x)$ we write $I(S)=I^{*}(S)+E^{*}(S)$ where

$$
\begin{aligned}
& I^{*}(S)=\int_{A}\left\{\left[f\left(x, z, p_{0}\right)-p_{0}^{i} f_{p^{i}}\left(x, z, p_{0}\right)\right]+p^{i} f_{p^{i}}\left(x, z, p_{0}\right)\right\} d x, \\
& E^{*}(S)=\int_{A} E\left(x, z, p_{0}, p\right) d x .
\end{aligned}
$$

Lemma 3.6. Let $S_{0}$ satisfy the conditions of Lemma 3.1. Let $\left\{S_{\alpha}\right\}(\alpha=1,2$, ...) be a sequence of admissible surfaces with the following properties: (i) $S_{\alpha}$ coincides with $S_{0}$ on $C$; (ii) $z_{\alpha}$ approaches $z_{0}$ uniformly on $\bar{A}$ as $\alpha \rightarrow \infty$; (iii) for almost all $x$ in $A, p_{\alpha}^{i} p_{\alpha}^{i}<M$ ( $\alpha$ not summed); (iv) $\lim \sup _{\alpha \rightarrow \infty} I\left(S_{\alpha}\right) \leqq I\left(S_{0}\right)$. Then $\lim _{\alpha \rightarrow \infty} I\left(S_{\alpha}\right)=I\left(S_{0}\right)$, and

$$
\lim _{\alpha \rightarrow \infty} E^{*}\left(S_{\alpha}\right)=0 .
$$

Proof. Since $E^{*}\left(S_{0}\right)=0$ and $I\left(S_{0}\right)=I^{*}\left(S_{0}\right)$,

$$
I\left(S_{\alpha}\right)-I\left(S_{0}\right)=I^{*}\left(S_{\alpha}\right)-I^{*}\left(S_{0}\right)+E^{*}\left(S_{\alpha}\right) .
$$

From Lemma 3.5, $I^{*}\left(S_{\alpha}\right)-I^{*}\left(S_{0}\right) \rightarrow 0$. By condition $\operatorname{II}_{N ; M}, E^{*}\left(S_{\alpha}\right) \geqq 0$ for $\alpha$ sufficiently large. Thus

$$
0 \geqq \lim \sup \left[I\left(S_{\alpha}\right)-I\left(S_{0}\right)\right]=\lim \sup E^{*}\left(S_{\alpha}\right) \geqq \lim \inf E^{*}\left(S_{\alpha}\right) \geqq 0 .
$$

Hence $\lim E^{*}\left(S_{\alpha}\right)=0$. From this it follows that $I\left(S_{\alpha}\right) \rightarrow I\left(S_{0}\right)$.

4. Proof of main theorem. Suppose the theorem is false. Then there exists a sequence of admissible surfaces $S_{\alpha}: z=z_{\alpha}(x)(\alpha=1,2,3, \cdots)$ such that $S_{\alpha}$ coincides with $S_{0}$ on $C, S_{\alpha}$ is not identical with $S_{0}, z_{\alpha} \rightarrow z_{0}$ uniformly on $\bar{A}, p_{\alpha}^{i} p_{\alpha}^{i} \equiv z_{\alpha x^{i} z_{\alpha x^{i}}<M}<(\alpha$ not summed) almost everywhere on $A$, and

$$
I\left(S_{\alpha}\right)-I\left(S_{0}\right) \leqq \min \left\{1 / \alpha, 1 / \alpha\left(\left\|z_{\alpha}-z_{0}\right\|^{2}+\left\|p_{\alpha}^{i}-p_{0}^{i}\right\| \cdot\left\|p_{\alpha}^{i}-p_{0}^{i}\right\|\right)\right\}
$$

( $\alpha$ not summed).

The index $\alpha$ will appear frequently in repeated form in the following discussion and we agree from the outset that such repetition is not to indicate summation on $\alpha$.

We let

$$
k_{\alpha}^{2}=\int_{A}\left[\left(z_{\alpha}-z_{0}\right)^{2}+\left(p_{\alpha}^{i}-p_{0}^{i}\right)\left(p_{\alpha}^{i}-p_{0}^{i}\right)\right] d x .
$$

Since $S_{\alpha}$ is distinct from $S_{0}$, we have $k_{\alpha}>0$. We define a sequence of admis- 
sible variations $\zeta_{\alpha}$ by

$$
\zeta_{\alpha}=\left(1 / k_{\alpha}\right)\left(z_{\alpha}-z_{0}\right)
$$

From (4.2),

$$
\int_{\Lambda}\left(\zeta_{\alpha}^{2}+\pi_{\alpha}^{i} \pi_{\alpha}^{i}\right) d x=1 \quad(\alpha=1,2,3, \cdots)
$$

where

$$
\boldsymbol{\pi}_{\alpha}^{i}=\zeta_{\alpha x^{i}}
$$

It follows from Theorem 2.1 that for a subsequence $\left\{\zeta_{\alpha}\right\}$, designated by the same subscript, we have functions $\zeta_{0}, \pi_{0}^{3}$ which are in $L_{2}$ on $\bar{A}$ such that $\left\|\zeta_{\alpha}-\zeta_{0}\right\| \rightarrow 0$, and $\pi_{0}^{i}$ satisfy

$$
\int_{0}^{i} V^{i}(x) \pi_{\alpha}^{i} d x \rightarrow \int_{0}^{i} V_{0}^{i} d x
$$

for $V^{i}$ arbitrary functions in $L_{2}$ on $\bar{A}$, and $e$ an arbitrary measurable subset of $\bar{A}$. We restrict ourselves to this subsequence henceforth. Also there exists a sequence $\left\{\xi_{\alpha}\right\}$ of admissible variations such that

$$
\left\|\xi_{\alpha}-\zeta_{0}\right\|^{2}+\left\|\kappa_{\alpha}^{i}-\pi_{0}^{i}\right\| \cdot\left\|\kappa_{\alpha}^{i}-\pi_{0}^{i}\right\| \rightarrow 0
$$

where $\kappa_{\alpha}^{i}=\xi_{\alpha x}$.

As before, we may write

$$
I(S)=I^{*}(S)+E^{*}(S)
$$

where $I^{*}, E^{*}$ are defined in equation (3.1). Thus

(4.4) $\left(1 / k_{\alpha}^{2}\right)\left\{I\left(S_{\alpha}\right)-I\left(S_{0}\right)\right\}=\left(1 / k_{\alpha}^{2}\right)\left\{I^{*}\left(S_{\alpha}\right)-I^{*}\left(S_{0}\right)\right\}+\left(1 / k_{\alpha}^{2}\right) E^{*}\left(S_{\alpha}\right)$.

LEMMA 4.1. For the sequence of surfaces $\left\{S_{\alpha}\right\}$ described above,

$$
\lim _{\alpha \rightarrow \infty} \frac{1}{k_{\alpha}^{2}}\left\{I^{*}\left(S_{\alpha}\right)-I^{*}\left(S_{0}\right)\right\}=\frac{1}{2} \int_{A}\left(f_{z z} \zeta_{0}^{2}+2 f_{z p^{i}} \zeta_{0} \pi_{0}^{i}\right) d x .
$$

Proof. From the definition of $I^{*}$,

$$
\begin{aligned}
\frac{1}{k_{\alpha}^{2}}\left\{I^{*}\left(S_{\alpha}\right)-\right. & \left.I^{*}\left(S_{0}\right)\right\} \\
& =\frac{1}{k_{\alpha}^{2}} \int_{A}\left\{f\left(x, z_{\alpha}, p_{0}\right)-f\left(x, z_{0}, p_{0}\right)+\left(p_{\alpha}^{i}-p_{0}^{i}\right) f_{p^{i}}\left(x, z_{\alpha}, p_{0}\right)\right\} d x \\
& =\frac{1}{k_{\alpha}} I_{1}\left(\zeta_{\alpha}\right)+\frac{1}{2} \int_{\Lambda}\left\{U_{\alpha}(x) \zeta_{\alpha}^{2}+2 V_{\alpha}^{i}(x) \zeta_{\alpha} \pi_{\alpha}^{i}\right\} d x,
\end{aligned}
$$


where $U_{\alpha}, V_{\alpha}^{\alpha} \rightarrow f_{z z}\left(x, z_{0}, p_{0}\right), f_{z p^{i}}\left(x, z_{0}, p_{0}\right)$ uniformly on $A$ by Taylor's remainder theorem. Since $S_{0}$ is an extremaloid, we have $I_{1}\left(\zeta_{\alpha}\right)=0$ and, rewriting the second integral,

$$
\begin{aligned}
\frac{1}{k_{\alpha}^{2}}\left\{I^{*}\left(S_{\alpha}\right)-I^{*}\left(S_{0}\right)\right\}= & \frac{1}{2} \int_{\Lambda} \zeta_{0}\left\{\left(U_{\alpha}-f_{z z}\right) \zeta_{\alpha}+2\left(V_{\alpha}^{i}-f_{z p^{p}}\right) \pi_{\alpha}^{i}\right\} d x \\
& +\frac{1}{2} \int_{\Lambda}\left(\zeta_{\alpha}-\zeta_{0}\right)\left(U_{\alpha} \zeta_{\alpha}+2 V_{\alpha}^{i} \pi_{\alpha}^{i}\right) d x \\
& +\frac{1}{2} \int_{A}\left(f_{z z} \zeta_{0} \zeta_{\alpha}+2 f_{z p^{i}} \zeta 0 \pi_{\alpha}^{i}\right) d x .
\end{aligned}
$$

From the Schwarz inequality, equation (4.3), and the limit properties of $\zeta_{0}, \pi_{0}^{i}$, we obtain that the first two integrals on the right tend to zero while the third approaches the desired limit.

From Lemma 3.4 we obtain by integration

$$
E^{*}\left(S_{\alpha}\right) \geqq \lambda \int_{\Lambda}\left(p_{\alpha}^{i}-p_{0}^{i}\right)\left(p_{\alpha}^{i}-p_{0}^{i}\right) d x
$$

By Lemma 3.6, $E^{*}\left(S_{\alpha}\right) \rightarrow 0$. Thus the integral on the right of (4.5) tends to zero. It follows, from a well known property of Lebesgue integration, that we may select a subsequence $\left\{S_{\alpha}\right\}$, denoted by the same subscript, such that for each $i, p_{\alpha}^{i} \rightarrow p_{0}^{i}$ almost uniformly on $A$. We restrict ourselves to this subsequence henceforth.

LeMma 4.2. For the sequence of surfaces described above,

$$
\liminf _{\alpha \rightarrow \infty} \frac{1}{k_{\alpha}^{2}} E^{*}\left(S_{\alpha}\right) \geqq \frac{1}{2} \int_{\Lambda} f_{p^{i} p^{i}}\left(x, z_{0}, p_{0}\right) \pi_{0} \pi_{0}^{j} d x .
$$

Proof. Let $e$ be a measurable subset of $A$ on which $p_{\alpha}^{4} \rightarrow p_{0}^{i}$ uniformly. By Taylor's remainder theorem

$$
\frac{1}{k_{\alpha}^{2}} \int_{0} E\left(x, z_{\alpha}, p_{0}, p_{\alpha}\right) d x=\frac{1}{2} \int_{0} U_{\alpha}^{i j}(x) \pi_{\alpha}^{i} \pi_{\alpha}^{j} d x
$$

where $U_{\alpha}^{\text {vi } \rightarrow f_{p^{i}} p^{i}}\left(x, z_{0}, p_{0}\right)$ uniformly on $e$. Thus

$$
\begin{aligned}
& \frac{1}{k_{\alpha}^{2}} \int_{0} E d x=\frac{1}{2} \int_{0}\left(U_{\alpha}^{i j}-f_{p^{i} p^{i}}\right) \pi_{\alpha}^{i} \pi_{\alpha}^{j} d x+\frac{1}{2} \int_{0} f_{p^{i} p^{i} \pi_{\alpha}^{i} \pi_{\alpha}^{j}} d x \\
& =\frac{1}{2} \int_{e}\left(U_{\alpha}^{i j}-f_{p^{i} p^{j}}\right) \pi_{\alpha}^{i} \pi_{\alpha}^{j} d x+\frac{1}{2} \int_{e} f_{p^{i} p^{j}}\left(\pi_{\alpha}^{i}-\pi_{0}^{i}\right)\left(\pi_{\alpha}^{j}-\pi_{0}^{j}\right) d x \\
& +\int_{e} f_{p^{s} p^{i}} \pi_{0}^{i} \pi_{\alpha}^{j} d x-\frac{1}{2} \int_{e^{i}} f_{p^{i} p^{i} \pi_{0}^{i} \pi_{0}^{i}}^{i} d x
\end{aligned}
$$


The first integral on the right approaches zero. The second is non-negative by Lemma 3.1. Thus in the limit we obtain

$$
\liminf _{\alpha \rightarrow \infty} \frac{1}{k_{\alpha}^{2}} \int_{e} E d x \geqq \frac{1}{2} \int_{e^{\prime}} f_{p^{i} p^{i} \pi_{0}^{i} \pi_{0}^{i} d x}
$$

From the non-negativeness of $E$, the region of integration on the left may be replaced by $A$. Since the measure of $e$ may be taken arbitrarily close to the measure of $A$, it follows from the absolute continuity of the integral on the right as a set function that the region $e$ may be replaced by $A$ on the right also. This completes the proof.

From equations (4.1) and (4.4),

$$
(1 / \alpha) \geqq\left(1 / k_{\alpha}^{2}\right)\left\{I\left(S_{\alpha}\right)-I\left(S_{0}\right)\right\}=\left(1 / k_{\alpha}^{2}\right)\left\{I^{*}\left(S_{\alpha}\right)-I^{*}\left(S_{0}\right)\right\}+\left(1 / k_{\alpha}^{2}\right) E^{*}\left(S_{\alpha}\right) .
$$

From Lemma 4.1,

$$
0 \geqq \frac{1}{2} \int_{A}\left(f_{z z} \zeta_{0}^{2}+2 f_{\left.z p^{i} \zeta_{0} \pi_{0}^{i}\right)}\right) d x+\limsup _{\alpha \rightarrow \infty} \frac{1}{k_{\alpha}^{2}} E^{*}\left(S_{\alpha}\right)
$$

From Lemma 4.2,

$$
I_{2}\left(\zeta_{0}, \pi_{0}\right) \leqq 0
$$

where the integral on the left is to be interpreted as the result of substituting the arguments $\zeta_{0}, \pi_{0}^{\boldsymbol{s}}$ for an admissible variation and its derivatives in the second variation.

Consider now the sequence of admissible variations $\left\{\xi_{\alpha}\right\}$. By the strengthened Jacobi condition we have

$$
I_{2}\left(\xi_{\alpha}\right) \geqq \gamma\left\|\xi_{\alpha}\right\|^{2} .
$$

Since $\left(\xi_{\alpha}, \kappa_{\alpha}\right)$ converges to $\left(\zeta_{0}, \pi_{0}\right)$ in the mean of order two on $A$ we obtain in the limit

$$
I_{2}\left(\zeta_{0}, \pi_{0}\right) \geqq \gamma\left\|\zeta_{0}\right\|^{2}
$$

It is clear, by comparison with (4.7), that we have a contradiction, and hence the proof of the theorem if we can show that $\left\|\zeta_{0}\right\|^{2} \neq 0$.

Suppose $\left\|\zeta_{0}\right\|=0$. Then $\zeta_{0}$ would be zero almost everywhere on $A$, and from equation (4.6) we should have

$$
\left(1 / k_{\alpha}^{2}\right) E^{*}\left(S_{\alpha}\right) \rightarrow 0 .
$$

Dividing both sides of (4.5) by $k_{\alpha}^{2}$ and taking the limit we should have

$$
\int_{\Lambda}^{i} \pi_{\alpha}^{i} \pi_{\alpha}^{i} d x \rightarrow 0
$$


On the other hand, from equation (4.3) and $\left\|\zeta_{\alpha}-\zeta_{0}\right\| \rightarrow 0$ we have

$$
\int_{A} \pi_{\alpha}^{i} \pi_{\alpha}^{i} d x \rightarrow 1,
$$

which yields the desired contradiction.

\section{REFERENCES}

1. S. Banach, Théorie des operations linéaires, Warsaw, 1932.

2. A. B. Carson, An analogue of Green's theorem for multiple integral problems in the calculus of variations, Dissertation, University of Chicago, 1942.

3. M. R. Hestenes, Extension of the range of a differentiable function, Duke Math. J. vol. 8 (1941) pp. 183-192.

4. - Sufficient conditions for the isoperimetric problem of Bolza in the calculus of variations, Trans. Amer. Math. Soc. vol. 60 (1946) pp. 93-118.

5. L. Lichtenstein, Untersuchungen uber zweidimensionale reguläre variationsprobleme, Monatshefte für Mathematik und Physik vol 28 (1917) pp. 3-51.

6. E. J. McShane, Sufficient conditions for a weak relative minimum in the calculus of variations, Trans. Amer. Math. Soc. vol. 52 (1942) pp. 344-379.

7. C. B. Morrey, Jr., Functions of several variables and absolute continuity, II, Duke Math. J. vol. 6 (1940) pp. 187-215.

8. F. G. Myers, Sufficient conditions for the problem of Lagrange, Duke Math. J. vol. 10 (1943) pp. 73-97.

9. W. T. Reid, The Jacobi condition for the double integral problem of the calculus of variations, Duke Math. J. vol. 5 (1939) pp. 856-870.

10. - Sufficient conditions by expansion methods. for multiple integral problems of the calculus of variations, Bull. Amer. Math. Soc. vol. 45 (1939) p. 679.

University of Chicago,

Chicago, Ill. 